Vol. 23 - No. 13 - April $5 \cdot 2013$

www.afm-journal.de

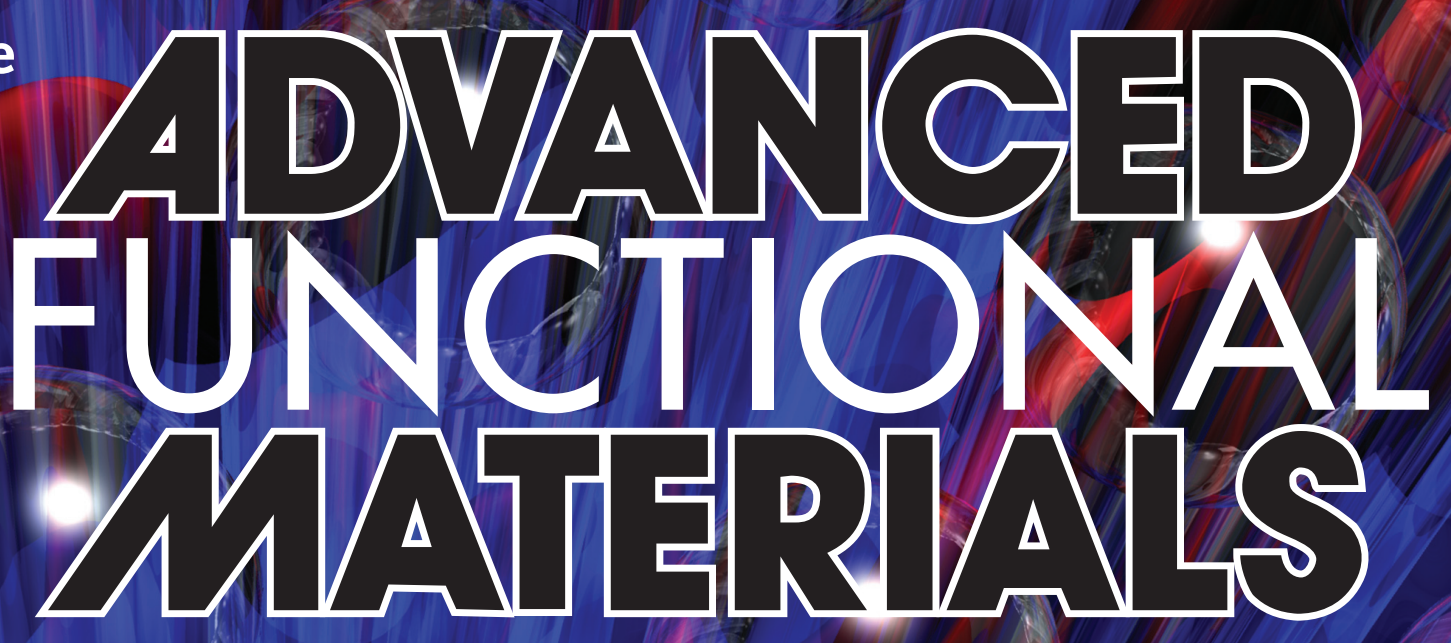

8
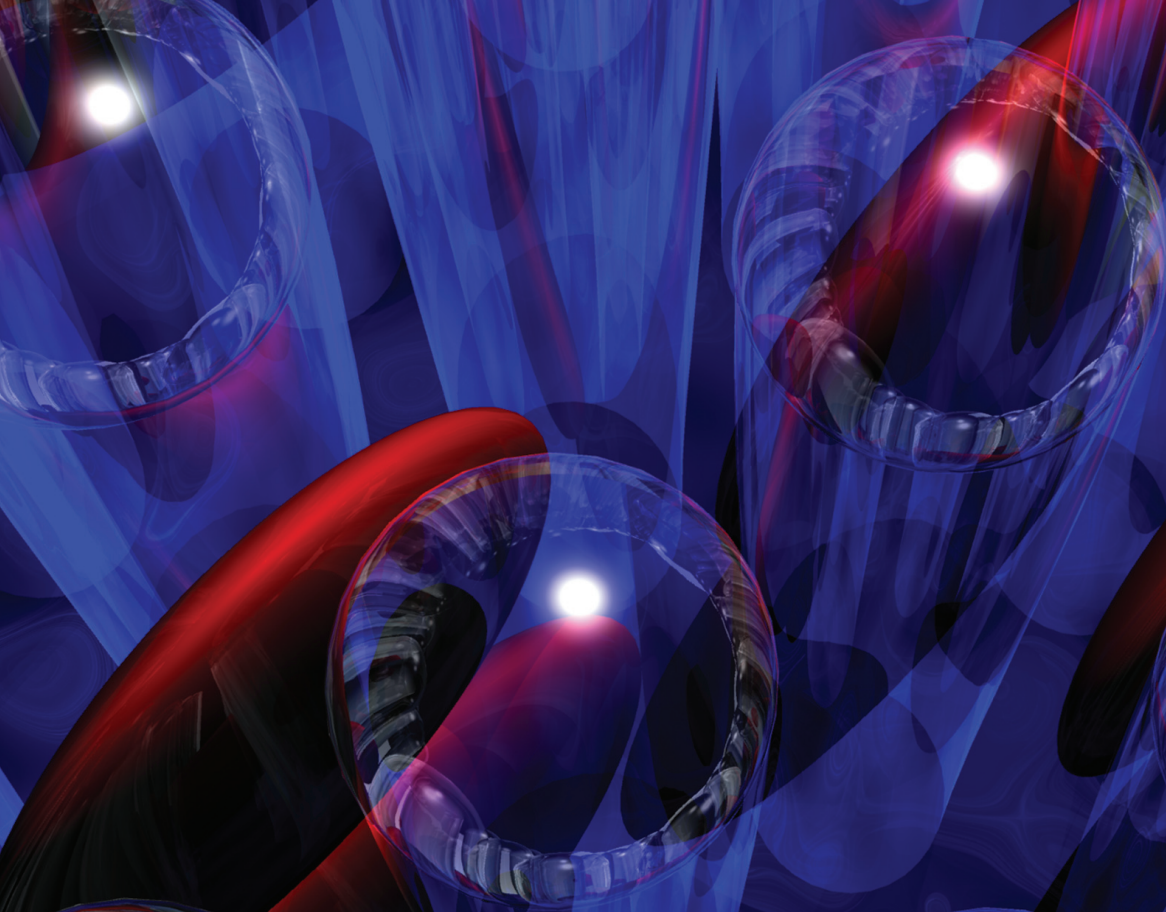

WILEY-VCH
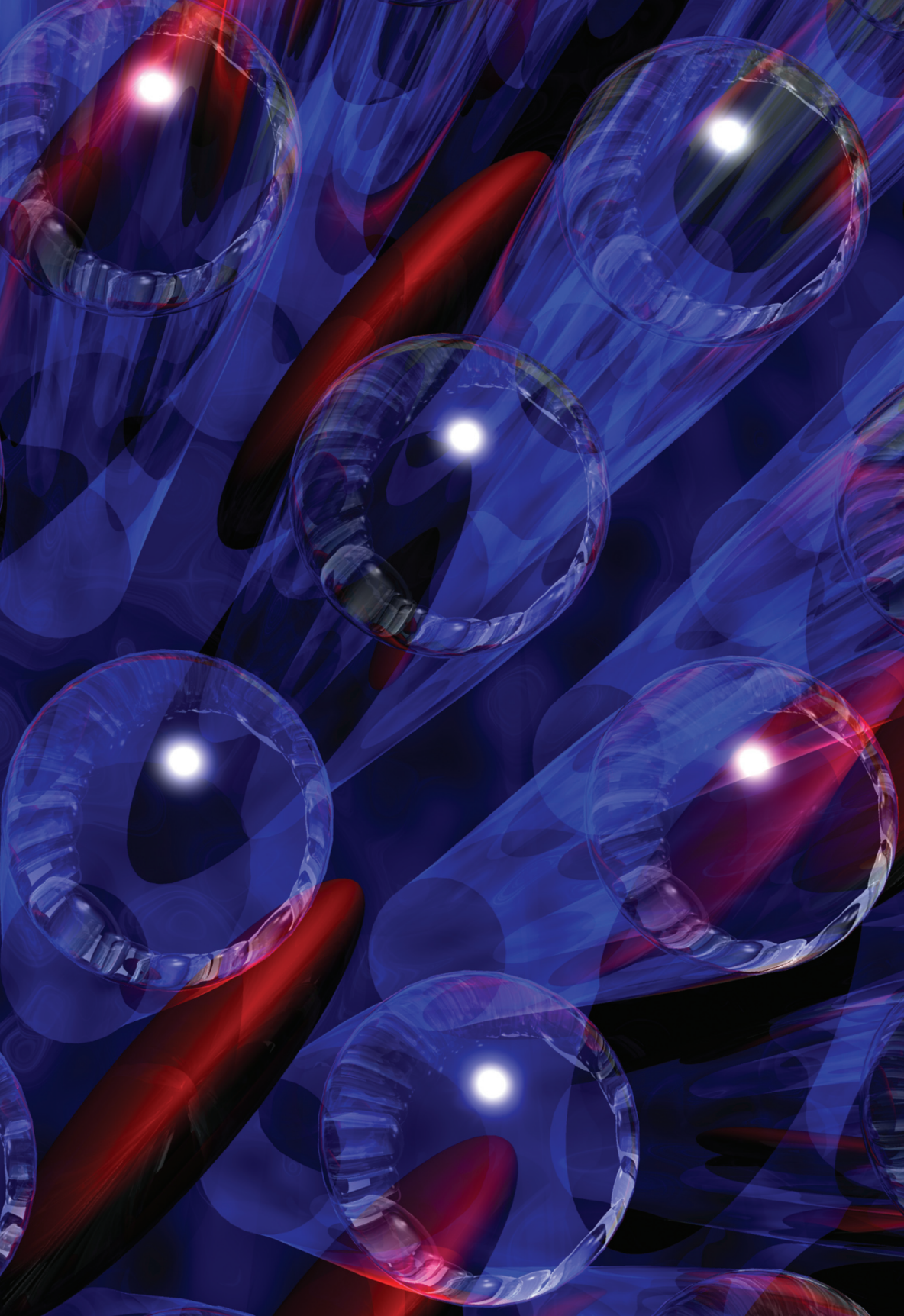


\title{
Tuning Dichroic Plasmon Resonance Modes of Gold Nanoparticles in Optical Thin Films
}

\author{
Lola Gonzalez-García, Julian Parra-Barranco, Juan Ramón Sanchez-Valencia, Javier Ferrer, \\ Mari-Cruz Garcia-Gutierrez, Angel Barranco,* and Agustín R. Gonzalez-Elipe*
}

\begin{abstract}
A simple method is presented to tune the gold surface plasmon resonance (SPR) modes by growing anisotropic nanoparticles into transparent $\mathrm{SiO}_{2}$ thin films prepared by glancing angle deposition. In this type of composite film, the anisotropy of the gold nanoparticles, proved by gracing incidence small angle X-ray scattering, is determined by the tilted nanocolumnar structure of the $\mathrm{SiO}_{2}$ host and yields a strong film dichroism evidenced by a change from an intense colored to a nearly transparent aspect depending on light polarization and/or sample orientation. The formation in these films of lithographic non-dichroic SPR patterns by nanosecond laser writing demonstrates the potentialities of this procedure to develop novel optical encryption or anti-counterfeiting structures either at micrometer- or macroscales.
\end{abstract}

fabrication of dichroic filters, ${ }^{[4]}$ polarized light nanosources, ${ }^{[5]}$ and materials with second-order nonlinearities. ${ }^{[6]}$ A typical feature of these nanostructures is a macroscopic optical dichroism resulting from the excitation of two distinct SPR modes, one along their longest dimension (longitudinal mode, LonSPR) and another perpendicular to it (transverse mode, TrSPR).

A generalized method for the nanoscale fabrication of SPR structures involves a soft lithography induced by light or electron beams. Although this type of methods do provide an accurate control over the nanostructrure of metal assemblies, ${ }^{[7-11]}$ its use is hampered by their high cost and

\section{Introduction}

Surface plasmon resonance (SPR) is a confined electromagnetic wave at the surface of a nanometric conductor characterized by a wavelength dependence which is affected by the size, shape, dielectric environment and nature of the metal nanostructure. ${ }^{[1-3]}$ The growth of anisotropic metal assemblies or nanoparticles (NPs) with high aspect ratio geometries is a perfect example of the importance of shape to tune the SPR. Electromagnetic waves propagating along the surface of these nanostructures present different resonances depending on the orientation of the light electrical field vector with respect to their longest axis. This dependence has been exploited for the

L. Gonzalez-Garcia, J. Parra-Barranco,

Dr. J. R. Sanchez-Valencia, ${ }^{[+]}$Dr. A. Barranco,

Prof. A. R. Gonzalez-Elipe

Nanotechnology on Surfaces laboratory

Instituto de Ciencia de Materiales de Sevilla (CSIC-US)

Avda. Américo Vespucio 49, 41092 Sevilla, Spain

E-mail: angel.barranco@csic.es; arge@icmse.csic.es

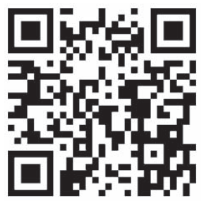

Dr. J. Ferrer

Centro Nacional de Aceleradores (CNA)

Universidad de Sevilla-CSIC

Avenida Thomas A. Edison 7, 41092 Sevilla, Spain

Dr. M.-C. Garcia-Gutierrez

Instituto de Estructura de la Materia (CSIC)

Serrano 121, 28006 Madrid, Spain

[+]Present address: EMPA, Swiss Federal Laboratories for

Materials Science and Technology, nanotech@surfaces Laboratory,

Überlandstrasse 129, 8600 Dübendorf, Switzerland

DOI: 10.1002/adfm.201201900 the long times required for manufacturing large areas. These limitations are circumvented in part by template-assisted synthesis methods using, for example, nanoporous alumina membranes to grow nanorods or nanotubes of metals with SPR. ${ }^{[12]}$ Related works have also reported alternative methods consisting of embedding in polymers arranged strips of spherical metal NPs ${ }^{[13]}$ or elongated metal NPs ${ }^{[14]}$ for the fabrication of optically anisotropic thin film filters.

Glancing angle deposited (GLAD) thin films prepared by evaporation present a highly porous nanostructure formed by tilted nanocolumns ${ }^{[15-18]}$ which, depending on the material, can aggregate in the form of linear "bundles" along the direction perpendicular to the incoming material flux. ${ }^{[19]}$ In previous works, we took advantage of this peculiar surface structure to prepare percolated silver nano-stripes on the surface of $\mathrm{SiO}_{2}$ GLAD thin films by a combined silver evaporation and laser treatment procedure. ${ }^{20,21]}$ However, although these unique porous characteristics make GLAD thin films good candidates as hosts for the template synthesis of composite materials, no systematic attempts have been reported about this application, except for their use to infiltrate active components of dyesensitized or hybrid solar cells. ${ }^{[22,23]}$ In the the present work, we present a completely new template procedure using polymers and chemically driven routes for the tailored synthesis of isolated gold nanoparticles (NPs) in the bulk of GLAD thin films acting as hosts. The method is simple, can be scaled up to large substrates and provides the possibility of tuning the SPR modes of gold NPs and their optical anisotropy. A peculiarity of the obtained films is that they present dichroism around both their azimuthal and polar axis, a feature that goes far beyond the current capabilities offered by the aforementioned procedures of NPs template synthesis ${ }^{[7-12]}$ or polymeric embedment 
aproaches. ${ }^{[13,14]}$ To assess the asymmetry and tilted arrangement of the gold NPs we use scanning electron microscopy (SEM) and grazing-incidence small-angle X-ray scattering (GISAXS). This latter technique, not utilized previously for the characterization of anisotropic composite thin films, has recently revealed as a powerful characterization method of thin films with tilted nanocolumns. ${ }^{[24]}$ Meanwhile, to clearly prove that the optical dichroism is due to the formation in the films of tilted and anysotropic gold NPs, optical simulations of the spectra have been carried out to clearly establish the dependence between optical properties and particle characteristics.

Besides the development of new synthesis and characterization approaches for preparing dichroic gold NPs, in the final part of this paper we prove that laser irradiation of the $\mathrm{Au} / \mathrm{SiO}_{2}$ films removes their dichroism in the illuminated zones. Relying on this result, we also show that a systematic application of
www.MaterialsViews.com

this technique in combination with a patterned deposition of the anisotropic GLAD films offers unprecedented possibilities for optical encryption or anti-counterfeiting applications not achievable with the current approaches using laser treatment of polymers with embedded NPs. ${ }^{[14]}$

\section{Results and Discussion}

\subsection{Tailored Formation of Gold Nanoparticles}

The preparation procedure of the gold NPs implies their molding in the intercolumnar space of the GLAD films. According to the scheme in Figure 1a, the experiment consists of the infiltration and reduction of a gold compound while it is dragged into

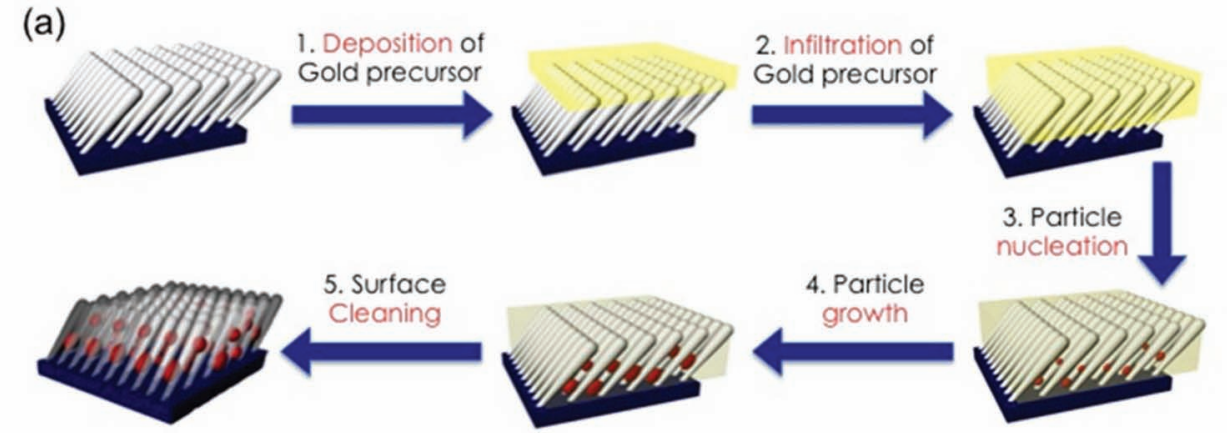

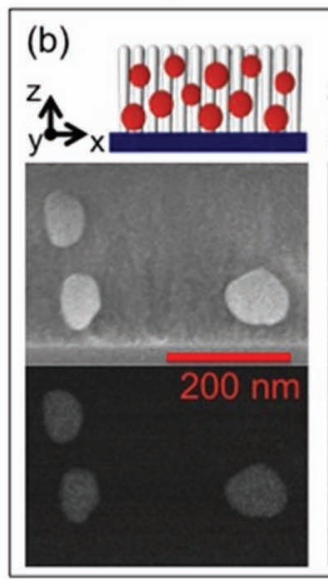

(d)

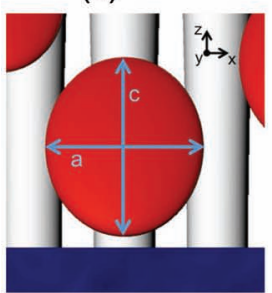

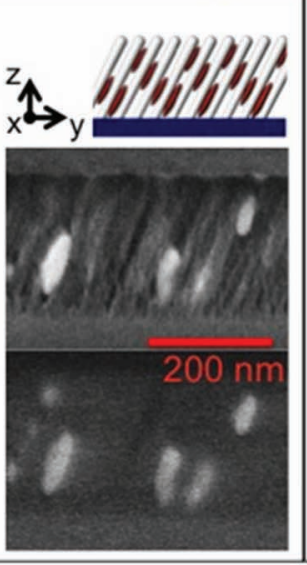

(c)
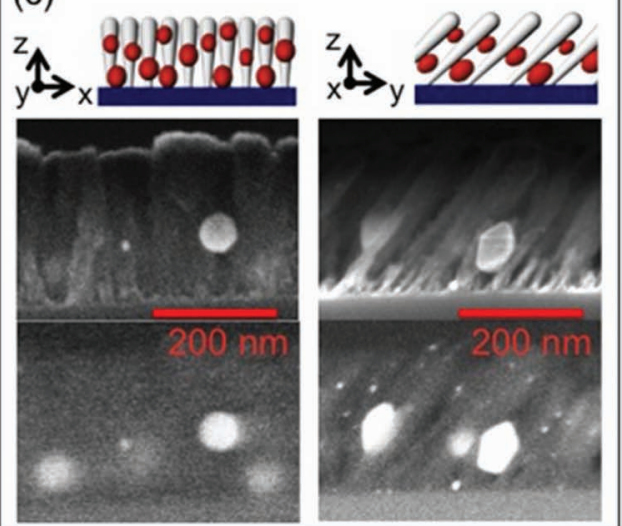

(f)
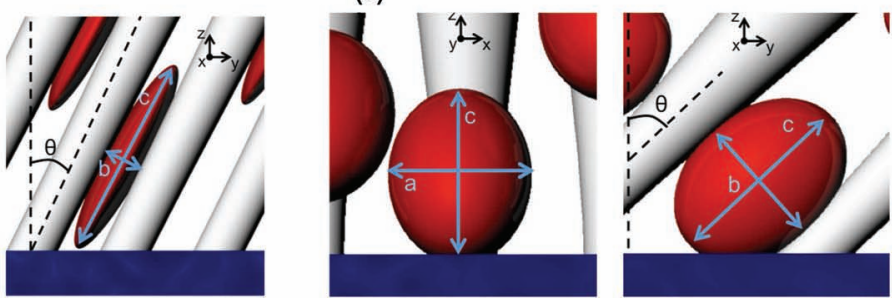

Figure 1. a) Scheme of the synthesis method to prepare anisotropic gold nanoparticles in $\mathrm{SiO}_{2}$ GLAD thin films: 1 . Deposition of a gold precursor together with PMMA on the film surface. 2. Infiltration of PMMA and precursor within the intercolumnar pores of the films. 3. Nucleation of gold particles and removal of PMMA. 4. Growth of gold nanoparticles. 5. Cleaning of the surface. b,c) Cross sectional SEM micrographs of Au/SiO $\mathrm{CLAD}_{2}$ thin films deposited at a) $60^{\circ}$ and b) $85^{\circ}$. Micrographs along the $x-z$ and $y-z$ orientation planes are reported in the figure as indicated, including secondary (top) and backscattered (bottom) electron images. 2D models with inserted coordinate axis are presented for a better understanding of the experimental results. d) Schematic representation of a NP along the $y$ - and $x$-directions in the $\mathrm{SiO}_{2}-60^{\circ}$ thin films with indication of its axis dimensions. f) Idem for $\mathrm{SiO}_{2}-85^{\circ}$ thin films. 
the pores by diffusion of PMMA. To tailor the shape, size, and orientation of gold NPs, we controlled the tilting angle of the host film by selecting their deposition angles $(\alpha)$ as reported in the Experimental Section and further discussed in ref. [25] and in the Supporting Information. The infiltrated amount of gold determined by Rutherford back scattering was around $2 \times$ $10^{16} \mathrm{~cm}^{-2}$ with a similar in-depth distribution irrespective of the type of film (see Figure S1 and Table S1 in the Supporting Information).

The size, shape, and orientation of the gold NPs were tightly dependent on the tilting angle and distance among the nanocolumns which, in turn, depend on the evaporation angle of the host $\mathrm{SiO}_{2}$ thin films. This is illustrated in Figure 1b,c showing the nanostructure of $\mathrm{Au} / \mathrm{SiO}_{2}$ thin films for hosts deposited at $\alpha=60^{\circ}$ and $85^{\circ}$, respectively. In these images it is apparent that the angle formed by the nanocolumns and the normal to the substrate $(\theta)$ increases with the deposition angle $(\alpha)$ from $18^{\circ}$ to $35^{\circ}$ for $\alpha=60^{\circ}$ and $85^{\circ}$ (see exact values for the other samples in Table S1 of the Supporting Information). ${ }^{[15-18,25]}$ This effect is controlled by the shadowing effects appearing along the flux direction $(\gamma)$ of the incoming $\mathrm{SiO}_{2}$ material and is responsible for the optical birefringence of this type of thin films. ${ }^{[26]}$ Meanwhile, in the perpendicular direction $(x)$, nanocolumns appear less separated due to a "bundling" effect that yields long void channels among successive lines of partially associated columns. ${ }^{[19]}$ Molding of the gold NPs (bright spots in Figure 1) in this intercolumnar space is clearly the reason for their tilting by the angle $\theta$ and their shaping control. Thus, from the two examples reported in Figure 1, it appears that the smaller average intercolumnar distance along the $\gamma$-direction in the $60^{\circ}$ $\mathrm{SiO}_{2}$ films constrains the gold NPs in a narrower space, so that they develop an elliptical shape in the $y-z$ plane and an aspect ratio close to unity along the $x-z$ plane (Figure $1 \mathrm{~b}$ ). This less restricted NP growth in the $x-z$ plane must be associated with the long percolated channels produced by the "bundling" association of nanocolumns. By contrast, the much higher separation between adjacent nanocolumns in the $y$-direction (Figure 1c) in sample $\mathrm{SiO}_{2}-85^{\circ}$ releases the growth restrictions in this and in the $x$ - (also in $z$-) directions, resulting in a quasispherical shape for the gold NPs. It is also apparent in Figure 1 that the longest dimension of the NPs decreases slightly when moving from the $\mathrm{SiO}_{2}-60^{\circ}$ to the $\mathrm{SiO}_{2}-85^{\circ}$ thin films. These changes in particle size and shape are schematically represented in Figure 1d,f, including a definition of the axis dimensions $(a, b, c)$ of the NPs. According to this scheme, in sample $\mathrm{SiO}_{2}-60^{\circ} \mathrm{a} \approx \mathrm{c}>\mathrm{b}$, while in sample $\mathrm{SiO}_{2}-85^{\circ}$ the three dimensions have similar values. In the former sample, the SEM images in Figure 1 also suggest that the a and $\mathrm{c}$ axis dimensions are slightly larger than in the latter. This first assessment of particle shapes is confirmed by simulation of the SPR reported in Sections 2.3 and 2.4.

\subsection{Proving the Asymmetry and Long Range Order of Gold NPS with GISAXS}

To confirm over large areas the anisotropic and tilted geometry of the gold NPs, their similar shape and size and their homogeneous distribution, we characterized the $\mathrm{Au} / \mathrm{SiO}_{2}$ films by GISAXS. In a recent publication, ${ }^{[2]}$ we have evidenced that the tilted microstructure of GLAD thin films renders a characteristic asymmetric pattern when the nanocolumns are placed perpendicular to the incident X-ray beam. Figure 2a shows the
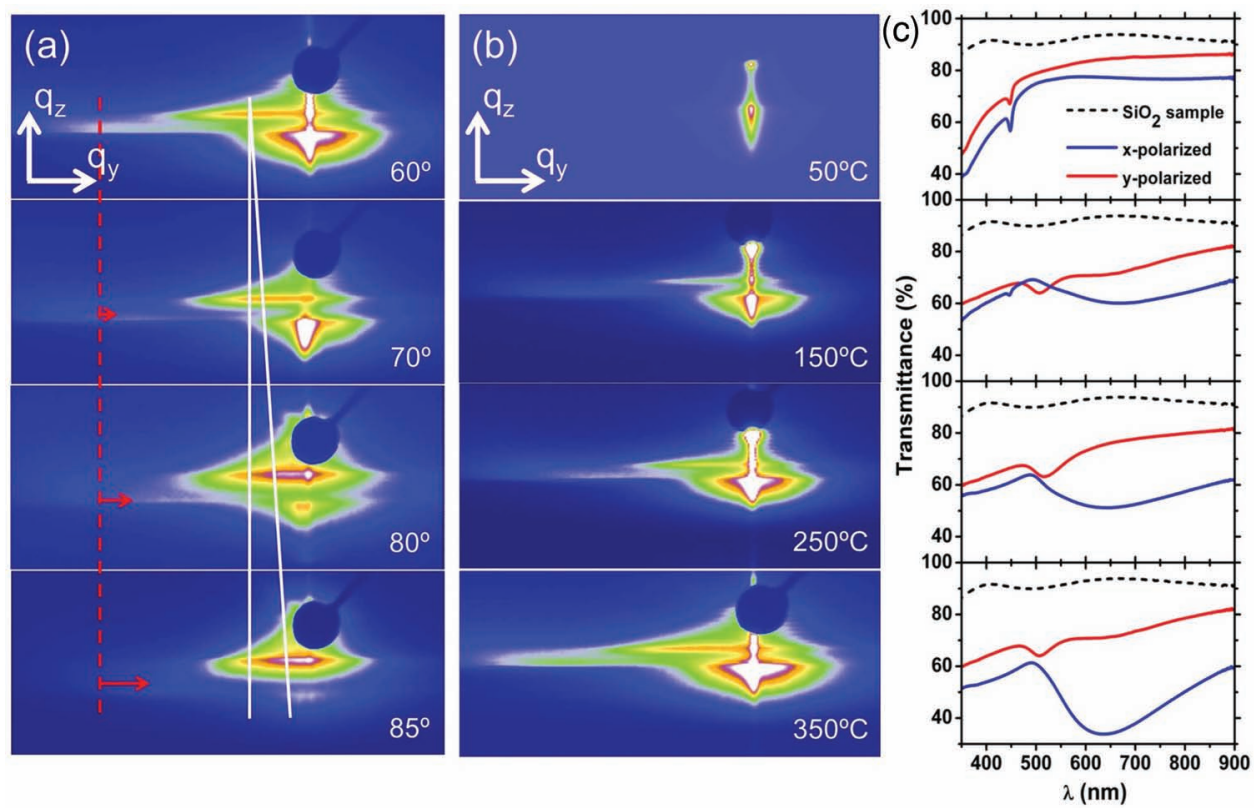

Figure 2. a) GISAXS patterns for $\mathrm{Au} / \mathrm{SiO}_{2} \mathrm{GLAD}$ thin films deposited at $\alpha=60^{\circ}, 70^{\circ}, 80^{\circ}$, and $85^{\circ}$. The red arrows point to the position where the maxima appear in equivalent $\mathrm{SiO}_{2}$ thin films without gold, while the white lines indicate the evolution in the position of the maximum in the patterns when going from the $60^{\circ}$ to the $85^{\circ}$ samples. b) In situ GISAXS measurements after heating at increasing temperatures reproducing the manufacture processing of $\mathrm{Au} / \mathrm{SiO}_{2} 60^{\circ} \mathrm{GLAD}$ thin films according to steps 3 and 4 in Figure 1a. c) $x$ - and $y$-polarized UV-vis light transmittance spectra of similar samples than in (b). 
GISAXS patterns of the investigated $\mathrm{Au} / \mathrm{SiO}_{2}$ composite thin films. By comparison with the patterns of bare $\mathrm{SiO}_{2}$ GLAD thin films (see Figure S2 in the Supporting Information and the position of red arrows in Figure 2 signaling the maxima in equivalent patterns of $\mathrm{SiO}_{2}$ ) it is apparent that the host material does not contribute to the diagrams, an effect that can be attributed to the much higher scattering cross-section of gold with respect to $\mathrm{SiO}_{2} \cdot{ }^{[27]}$ By discarding this contribution, the obtained asymmetric GISAXS patterns clearly confirm that the gold NPs are tilted and that therefore they have grown in the intercolumnar voids of the host. Furthermore, the development in the diagrams of well defined maxima proves that the distribution of gold NPs is homogenous, while the observed shift of the maxima towards the center of the patterns (see the white line pointing out the position of the maxima for the different diagrams) indicates that the correlation distance between particles increases with the deposition angle. In this regard, the decrease in pattern asymmetry with this parameter sustains a progressive equalization of particle dimensions when moving from sample $\mathrm{SiO}_{2}-60^{\circ}$ to sample $\mathrm{SiO}_{2}-85^{\circ}$. Although an insufficient definition of the maxima position precludes an accurate determination of geometrical dimensions, roughly calculated correlation distances were around $100 \mathrm{~nm}$, i.e., of the order of magnitude of the interparticular distances estimated when modeling the optical properties of the composite films (see Section 2.4).

On the other hand, GISAXS also proved to be an useful tool to monitor the formation of gold NPs during the preparation of the $\mathrm{Au} / \mathrm{SiO}_{2}$ thin films. Figure $2 \mathrm{~b}$ reports a series of selected GISAXS patterns obtained at the indicated temperatures by heating "in situ" the $\mathrm{Au} / \mathrm{SiO}_{2}-60^{\circ}$ precursor sample (i.e., steps 2 to 5 in Figure 1a). The optical changes occuring in parallel are reported in Figure 2c showing the polarized UV-vis transmittance spectra of equivalent samples subjected to similar heat treatments and then cooled down to room temperature for recording. GISAXS patterns do not depict any maximum for temperatures lower than $50{ }^{\circ} \mathrm{C}$, not even those expected for the $\mathrm{SiO}_{2}$ host (see Figure $\mathrm{S} 2$ in the Supporting Information) which must be disguised by the scattering effect of the gold atoms distributed within the PMMA. Consistently, the optical UV-vis spectrum of this sample does not depict any plasmon band, but an absorption peak at $450 \mathrm{~nm}$ attributed to molecularly distributed $\mathrm{HAuCl}_{4}$. A small difference in light transmission for both polarizations can be attributed to the inherent birefringence of the GLAD $\mathrm{SiO}_{2}$ thin films and the optical dispersive behavior of PMMA anisotropically distributed within the pores. The GISAXS patterns only developed an asymmetric maximum after heating at $150-250{ }^{\circ} \mathrm{C}$ and subsequently at $350{ }^{\circ} \mathrm{C}$ when it became quite intense. This experiment further supports that the asymmetric GISAXS patterns responds to the scattering of the X-rays in a quasi-periodic tilted arrangement formed by gold nanoellipsoids as previously observed by SEM. The development at temperatures higher than $150{ }^{\circ} \mathrm{C}$, with a maximum at $\approx 350{ }^{\circ} \mathrm{C}$, of a well defined SPR band in the UV-vis spectrum when recorded with $x$-polarized light (Figure 2c) confirms the attribution of the GISAXS maxima to a quasi-periodic tilted arrangement of gold nanoellipsoids. In the course of these experiments, we also proved the thermal stability of the films by performing heating experiments up to $500{ }^{\circ} \mathrm{C}$. After these heating experiments, neither the UV-vis nor the GISAXS spectra changed significantly, a feature that must be attributed to the restrictions imposed by the $\mathrm{SiO}_{2}$ nanocolumns to the diffusion of gold.

\subsection{Optical Anisotropy Around the Azimuthal Angle}

An optical anisotropy as that progressively developed by the precursor sample $\mathrm{Au} / \mathrm{SiO}_{2}-60^{\circ}$ heated at increasing temperatures (Figure 2c) was also observed for other composite thin films fabricated by using hosts prepared at different evaporation angles from $60^{\circ}$ to $85^{\circ}$. The $x$ - and $y$-polarized spectra in Figure 3 clearly show that the different composite films differ in the position of the LonSPR and TrSPR maxima of gold NPs. The difference between the position of the two SPR modes was maximum for sample $\mathrm{Au} / \mathrm{SiO}_{2}-60^{\circ}$ (i.e., 505 to $635 \mathrm{~nm}$ for the LonSPR and TrSPR, respectively) and progressively decreased with the deposition angle to reach a minimum for sample $\mathrm{Au} / \mathrm{SiO}_{2}-85^{\circ}$ (i.e., 525 to $550 \mathrm{~nm}$ for the LonSPR and TrSPR, respectively). Both in this sample and particularly in sample $\mathrm{Au} / \mathrm{SiO}_{2}$-PN (a GLAD film with perpendicular nanocolumns), the similar shape and position of the LonSPR and TrSPR bands indicate that the embedded gold NPs are quasi-spherical. As a consequence of the shift in the position of the SPR maxima for a given sample, by eye observation the $\mathrm{Au} / \mathrm{SiO}_{2}$ films presented different colors when illuminated with $x$ - and $y$-linearly polarized white light (Figure 3 (right)). Differences in color were maximum in sample $\mathrm{Au} / \mathrm{SiO}_{2}-60^{\circ}$ presenting blue and pink colors for these two polarizations, while color differences attenuated with the deposition angle and were negligible for sample $\mathrm{Au} / \mathrm{SiO}_{2}-\mathrm{PN}$. Equivalent responses could be obtained by azimuthally rotating the samples when illuminated with a fixed linearly polarized source of white light.

\subsection{Simulation of the Optical Dichroism}

The optical dichroism depicted by the composite $\mathrm{Au} / \mathrm{SiO}_{2}$ samples must be linked to the anisotropy of the gold NPs hostsynthesized in the bulk of the films. To further assess this point, the optical behavior of the $60^{\circ}$ and $85^{\circ}$ samples was modeled with the discrete dipole approximation (DDA), ${ }^{[28-31]}$ a numerical method to solve Maxwell equations that cannot be handled analytically. Among the different procedures proposed in literature, DDA is by far the most widely employed for metallic particles in complex surrounding environments. This method provides a mean to accurately determine the scattering and absorption of light by targets of arbitrary shape and complex dielectric media. To model the spectra we adopted the following assumptions i) a tilted arrangement of ellipsoidal gold NPs along the nanocolumns angle $\theta$; ii) one particle dimension (b) equivalent to the nanocolumn separation and different than the two others $(\mathrm{b} \neq \mathrm{a}=\mathrm{c})$; iii) an equivalent volume for the anisotropic NPs but a different effective refractive index in the different films; iv) a repeated arrangement of NPs along the $x-y$ plane and only one particle along the $\mathrm{z}$ direction.

Simulations of the extinction cross sections of gold nanoellipsoids with different geometries and orientations have been 


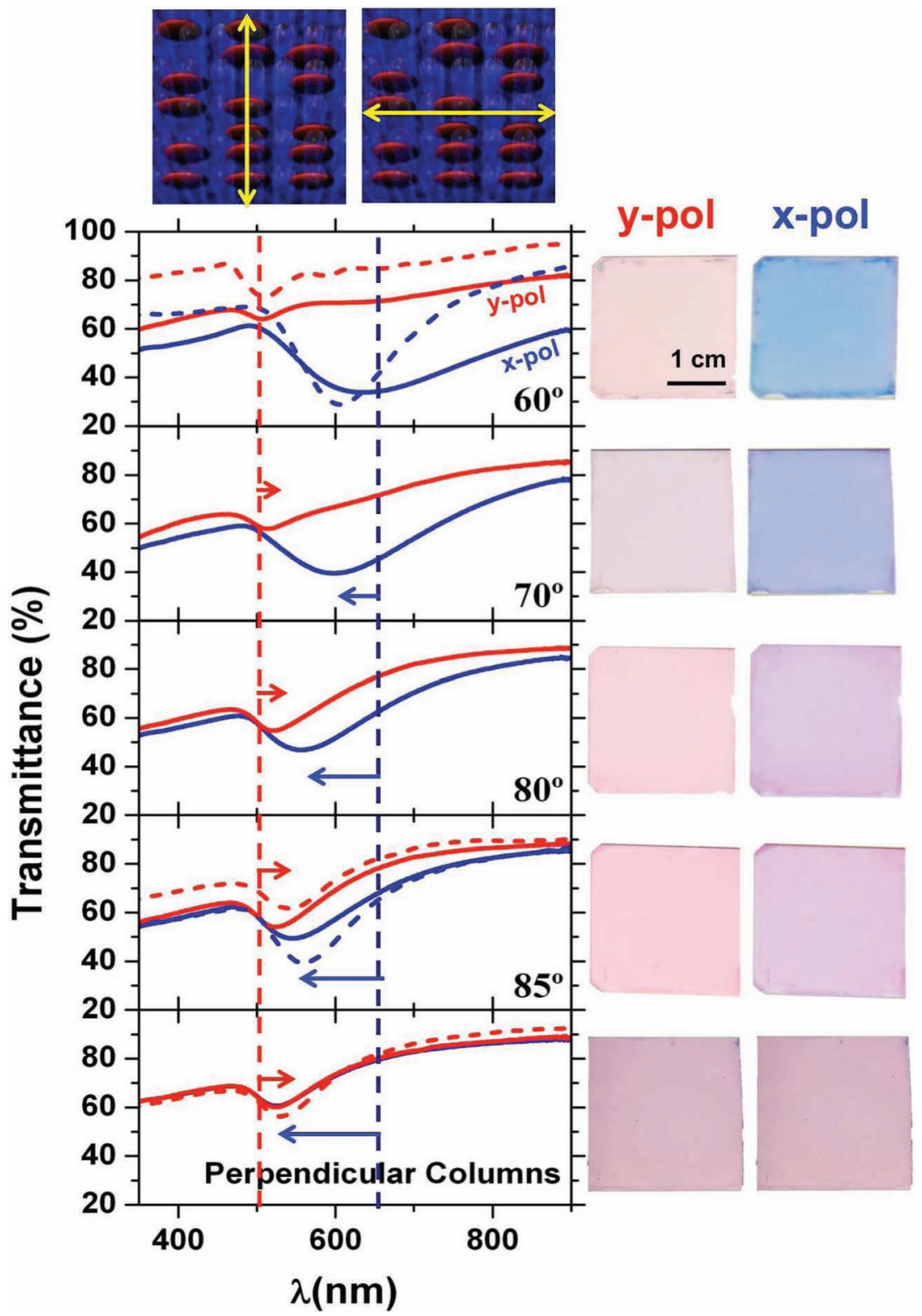

Figure 3. Full line: polarized UV-vis light transmittance spectra for $\mathrm{Au} / \mathrm{SiO}_{2} \mathrm{GLAD}$ thin films deposited at $\alpha=60^{\circ}, 70^{\circ}, 80^{\circ}$, and $85^{\circ}$ and a GLAD film consisting of perpendicular nanocolumns. Dashed line: selected simulated spectra (see text). The vertical dashed lines indicate the peak positions for sample $\mathrm{Au} / \mathrm{SiO}_{2}-60^{\circ}$, while the associated arrows indicate the relative shift found in the two SPR peaks of each sample. Pictures of samples illuminated with $x$ - and $y$-polarized white light are shown in the right side of the figure. The scheme at the top illustrates the orientation of the polarization vector of light with respect to a projection of the tilted NPs on the $x-y$ plane.

carried out by employing the code DDSCAT 7.1 developed by Draine and Flatau. ${ }^{[30-32]}$ To take into consideration the interaction with the surrounding NPs, in agreement with the SEM and GISAXS results, our model considers a periodic target of gold nanoellipsoids embedded in a medium with refractive index higher than that of the air. Figure 4a presents the geometrical model used for the DDA simulations where we have considered that gold nanoellipsoid geometry, orientation, periodicity, and dielectric environment are dependent on the $\mathrm{SiO}_{2}$ thin film deposition angle. The targets consist of $\mathrm{N}$ dipoles with their $\mathrm{N}_{x}, \mathrm{~N}_{\gamma}$ and $\mathrm{N}_{z}$ components aligned along the axes of nanoellipsoids that are surrounded by a medium with refractive index $\mathrm{n}_{\text {med }}$. A restriction of the DDA approximation is that the interdipole distance must satisfy the condition $|m| k s<1$, where $m$ is the complex refractive index of gold. For all the simulations this condition was set to be $|m| k s<0.1$, fulfilling by far the requirements of the method. It is important to note that the precision of the DDSCAT code increases with the number of dipoles, though at expenses of a significant increase in the computational time. Further details about the specific calculation procedure and the utilized formulations can be found in the Supporting information.

In the particular case of perpendicular columns, the rotation of the substrates during deposition produces not only a perpendicular growth of the $\mathrm{SiO}_{2}$ nanocolumns, but also that the intercolumnar separation is equal in the $x$ and $y$ directions. Because of that, the smallest axis of the nanoellipsoids (b) is not oriented anymore and these samples do not present optical dichroism. This situation was implemented in the simulations by using periodically arranged nanoellipsoids with the c-axis coincident with $z$ direction $(\theta=0)$, but with the $\mathrm{b}$-axis randomly oriented in the $x$-y plane.

The calculated transmittance spectra for both $x$ - and $\gamma$-polarizations are represented by dashed lines in Figure 3 where they are compared with the experimental results. This figure shows that under the assumptions of the calculations the simulated spectra were in good agreement with the experimental ones. Particle dimensions, tilting angles and NP separation in the $\mathrm{x}$ and $\mathrm{y}$ directions have been calculated for a big number of particles. Representative SEM pictures along $x-z$ and $\gamma-z$ planes for $\mathrm{Au} / \mathrm{SiO}_{2}-60^{\circ}$ and $-85^{\circ}$ are presented in Figure S3 of supporting information to assess the size, shape, tilting angle and separation between NPs. Average values calculated from these pictures are summarized in Table 1, clearly evidencing that while the number of elemental dipoles within the NPs does not significantly vary from one sample to another, their shape (differences between $\mathrm{a} / \mathrm{c}$ and $\mathrm{b}$ ) and dimensions (actual values of a, b, and c) do.

\subsection{Optical Anisotropy Around the Polar Angles}

Another striking optical feature of the investigated composite films, particularly evident for sample $\mathrm{Au} / \mathrm{SiO}_{2}-60^{\circ}$, was an 
(a)

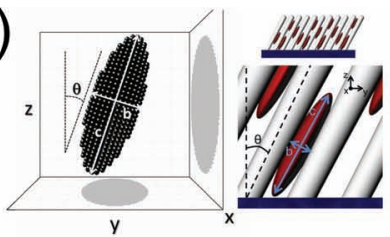

(b) Experimental

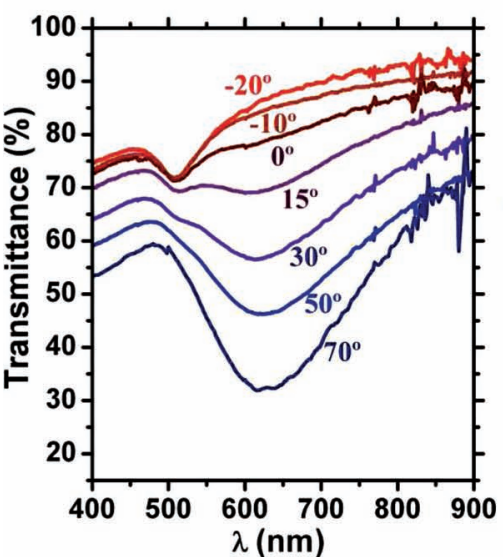

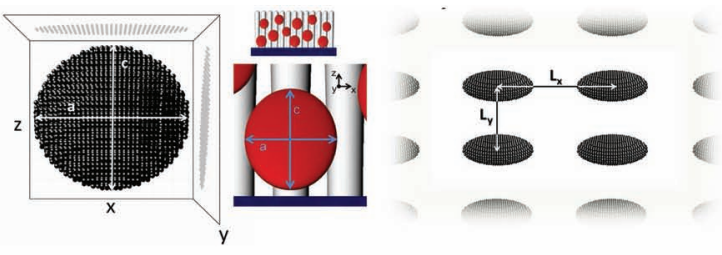

(c) Simulation
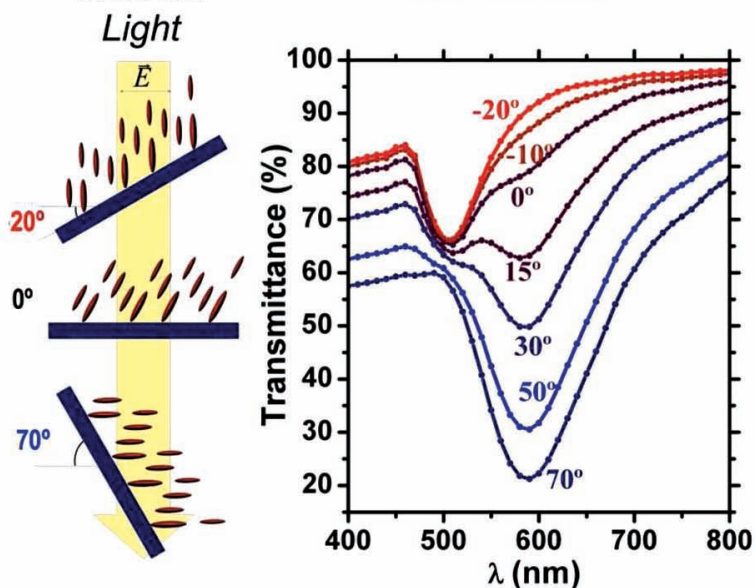

Figure 4. a) Schematic views of the simulated gold NPs. The black dots in the nanoellipsoids represent every polarizable point (dipoles) in a cubic lattice used in the program DDSCAT. Projection of the individual ellipsoids onto the $y-z$ and $x-z$ planes are presented to identify the geometry of the particle. The simulations were performed with nanoellipsoids distributed in the $x-y$ plane, and separated in the $x\left(L_{x}\right)$ and $y\left(L_{\gamma}\right)$ axis as presented in the scheme. b) $y$-polarized transmittance spectra of sample $\mathrm{Au} / \mathrm{SiO}_{2}-60^{\circ}$ as a function of the angle of rotation around the $x$-axis. The representation in the center shows how the rotation around $x$-axis aligns the long nanoparticle axis (with a maximum in ca. $+70^{\circ}$ ) or the short nanoparticle axis (with a maximum in ca. $-20^{\circ}$ ) with the incoming electric field light. As a result, turning the films around the $x$-axis produces the selective excitation of the $\operatorname{TrSPR}$ and LonSPR modes at $-20^{\circ}$ and $70^{\circ}$ orientation, respectively. c) Simulated $y$-polarized transmittance spectra evidencing a good agreement with the experimental spectra recorded at equivalent angles.

unusual change in transmittance upon rotation around their polar axis. For both $x$ - and $\psi$ - polarized lights, the position of the SPR peaks remained unaltered upon polar rotation around the $\gamma$-axis (only a small change in the SPR intensity was observed), but dramatically changed when using $\gamma$-polarized light and the sample was rotated around its $x$-axis. For sample $\mathrm{Au} / \mathrm{SiO}_{2}-60^{\circ}$, this behavior is illustrated in Figure 4 showing that by $x$-axis rotation to the left, the overall sample transmission for $\lambda>550 \mathrm{~nm}$ diminished and the peak at $505 \mathrm{~nm}$ sharpened. A maximum effect was found at approximately $-20^{\circ}$, coinciding with the tilting angle of the nanocolumns. Meanwhile, the $x$-axis rotation to the right produced the development of a wide peak at $\approx 635 \mathrm{~nm}$, which reached its maximum intensity at approximately $+70^{\circ}$. By visual inspection, this complete shift from the TrSPR (peak at $505 \mathrm{~nm}$ ) to the LonSPR (peak at $635 \mathrm{~nm}$ ) mode by $90^{\circ} x$-axis rotation rendered a pronounced change in color, an effect that was not observed by rotating around the $y$-axis. The schematic model in Figure $4 \mathrm{~b}$ explains graphically the observed effects. Furthermore, to account for the effect of rotation around the $x$-axis while illuminating with $y$-polarized light, DDA model simulations were carried out for sample $\mathrm{Au}$ / $\mathrm{SiO}_{2}-60^{\circ}$, taking from Table 1 the geometry and periodicity of gold nanoellipsoids as well as the dielectric characteristics of the medium (see further considerations in the Supporting Information). With respect to the previous calculations in Section 2.3 a difference here was that the NPs c axis was aligned along the $z$ direction $(\theta=0)$. This situation corresponds to NPs aligned with the the incoming light $(z)$, an orientation that is achieved experimentally by rotating the samples $-20^{\circ}$. To account for additional rotational effects, the local coordinate system of the targets was turned from 0 to $90^{\circ}$ (experimentally from -20 to 70 ) around the a axis of the NPs. The complete

Table 1. Parameters used for the simulations of the optical properties.

\begin{tabular}{|c|c|c|c|c|c|c|c|c|}
\hline Sample & $\mathrm{N}: \mathrm{N}_{x}, \mathrm{~N}_{\gamma}, \mathrm{N}_{z}$ & $\begin{array}{l}\mathrm{a}=\mathrm{c} \\
{[\mathrm{nm}]}\end{array}$ & $\begin{array}{c}b \\
{[\mathrm{~nm}]}\end{array}$ & Aspect ratio & $\begin{array}{c}\theta \\
{\left[{ }^{\circ}\right]}\end{array}$ & $\begin{array}{c}\mathrm{Lx} \\
{[\mathrm{nm}]}\end{array}$ & $\begin{array}{c}\text { Ly } \\
{[\mathrm{nm}]}\end{array}$ & $\mathrm{n}_{\text {media }}$ \\
\hline $\mathrm{Au} / \mathrm{SiO}_{2}-60$ & 6303: 33, 33, 11 & 120 & 40 & 3.0 & 18 & 360 & 120 & 1.4 \\
\hline $\mathrm{Au} / \mathrm{SiO}_{2}-85$ & $6588: 25,25,20$ & 90 & 72 & 1.2 & 35 & 185 & 185 & 1.2 \\
\hline $\mathrm{Au} / \mathrm{SiO}_{2}-\mathrm{PN}$ & $6588: 25,25,20$ & 90 & 72 & 1.2 & 0 & 185 & 185 & 1.2 \\
\hline
\end{tabular}


series of simulations as a function of rotation angle is presented in Figure 4c, where the labeled angles have been taken from the experiment. The good reproduction of the main features of the experimental spectra (i.e., peak position, shift, change in intensity) by the DDA simulations further supports that changes appearing upon polar angle rotation are due to both the anisotropy of NPs and their tilting orientation within the $\mathrm{SiO}_{2}$ nanocolumns.

\subsection{Laser Writing and Optical Encoding}

When the $\mathrm{Au} / \mathrm{SiO}_{2}-60^{\circ}$ samples were illuminated with enough power of a diode-pumped $\mathrm{Nd}$ :YAG laser emitting at $1064 \mathrm{~nm}$, their dichroism was removed and isotropic SPR bands could be seen in the spectra of the irradiated zones. This behavior suggests a reshaping of the gold nanoellipsoids and their transformation into spherical particles. Similar reshaping effects were reported by Liz-Marzan et al. ${ }^{[14]}$ for elongated gold NPs embedded in a polymer matrix, although in our case the tilted arrangement of NPs offers quite new and unprecedented patterning possibilities to encode information.

In our experiment, an excellent optical contrast with the non-written areas was also apparent by visual inspection when using x-polarized white light. By contrast, no laser patterns could be easily differentiated when the samples were examined with $\gamma$-polarized light. An example of this effect is reported in Figure $5 \mathrm{a}$ showing the $x$-polarized light laser patterns obtained with samples prepared at different evaporation angles $\alpha$. It is apparent that the contrast is progressively lost as $\alpha$ increases. The possibility of fine tuning the pattern profiles is evidenced in Figure $5 \mathrm{~b}$ showing a patterned barcode on a $\mathrm{Au} / \mathrm{SiO}_{2}-60^{\circ}$ sample that was only visible with x-polarized light. As evidenced by the enlarged images in Figure 5b, bar codes at the micron scale can be also written with this technique. In addition, we demonstrated that the magnitude of the dichroic response can be efficiently controlled by varying the laser output power. By modifying this parameter it was possible both to progressively control the magnitude of the changes in the UV-vis spectra recorded with $x$ - and $y$-polarized lights (see the spectra of the irradiated $\mathrm{Au}$ / $\mathrm{SiO}_{2}$ samples in Figure S4 in the Supporting Information) and to induce significant color changes as shown by the evolution of the written squares in Figure 5a.
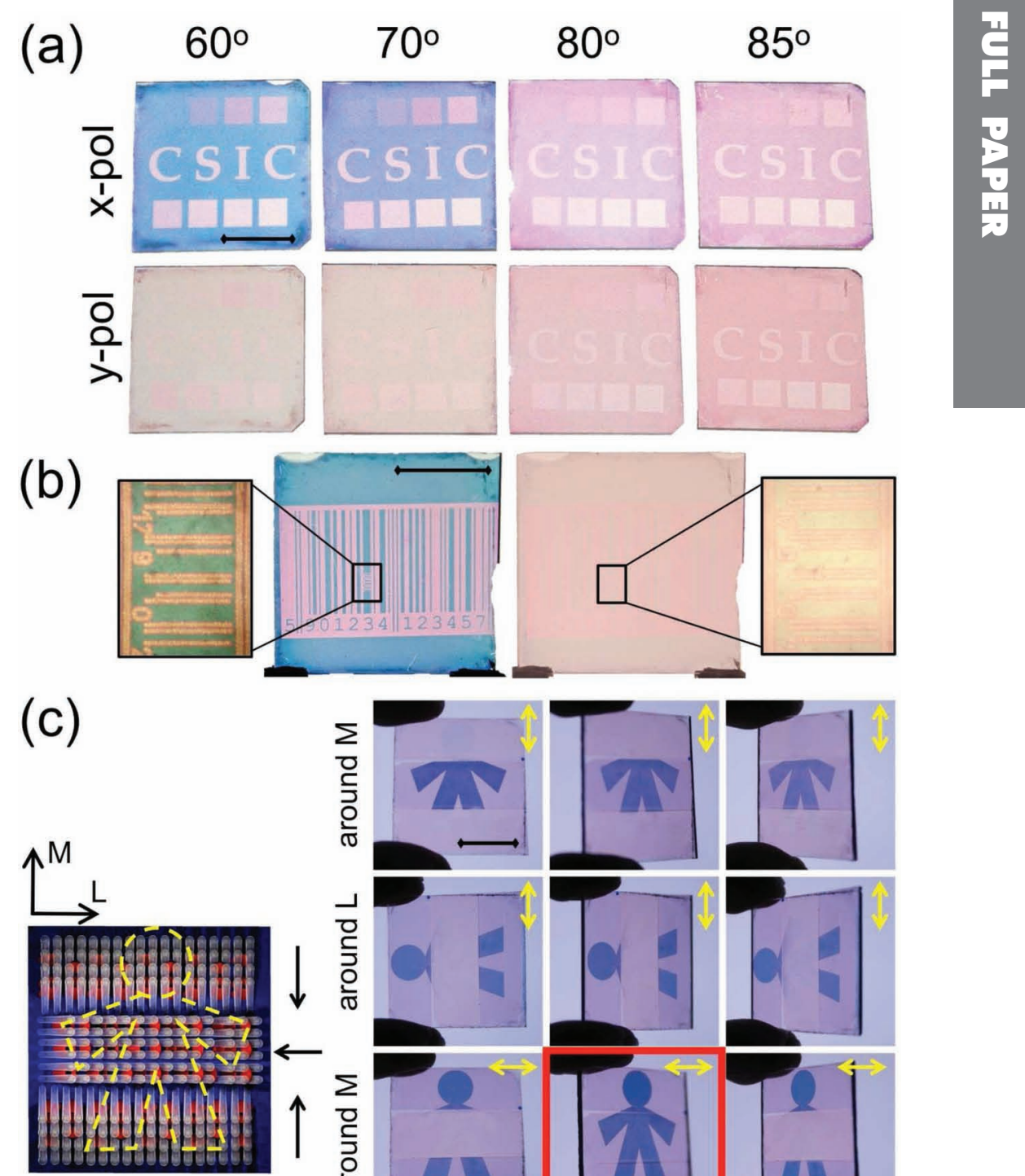

(c)
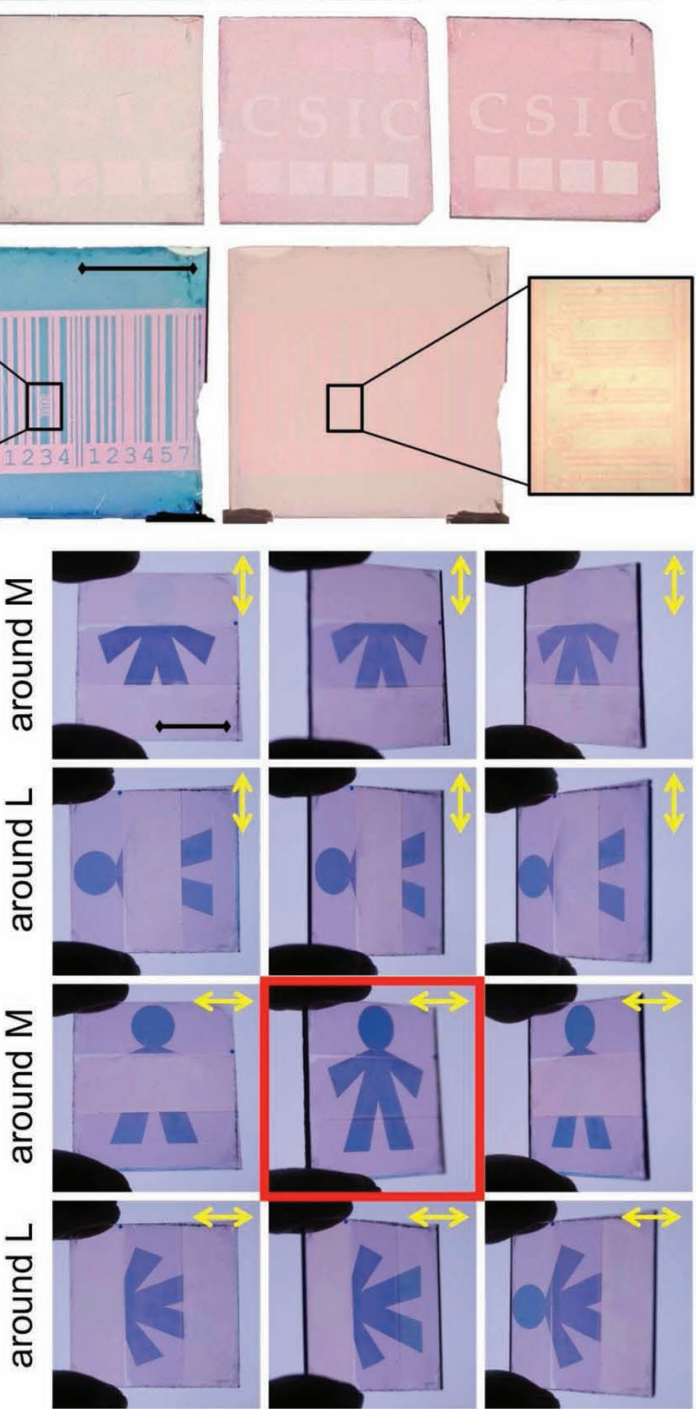

Figure 5. a) Pictures of a series of $\mathrm{Au} / \mathrm{SiO}_{2}$ samples deposited at $\alpha=60,70,80$, and $85^{\circ}$ illuminated with $x$ - and $y$-polarized light. Selected zones of these samples were treated with laser at powers ranging from 25 to $95 \%$ of the total output power $\left(240 \mathrm{~kW} / \mathrm{cm}^{-2}\right)$ at $10 \%$ intervals (eight squares in the outer zone of each sample). The central "C S I C" characters have been written by using a laser power of $60 \%$. b) Standard bar code written with the laser onto a $\mathrm{Au} /$ $\mathrm{SiO}_{2}-60^{\circ}$ sample and monitored with $x$ - and $y$-polarized lights. The zoom images show the presence of a secondary micrometric bar code engraved in one of the bars. c) Optical effects in an irradiated $\mathrm{Au} / \mathrm{SiO}_{2}-60^{\circ}$ sample with three different orientations of the nanocolumns. The scheme illustrates the different orientations of the gold nanoparticles in the original sample and the contour of a figure written by laser irradiation. The photographs on the right correspond to the irradiated sample illuminated with $x$ - (top figures) and $y$ - (bottom images) polarized lights and turned by $90^{\circ}$ around the axis perpendicular to the film and by approximately $\pm 45^{\circ}$ around the axis in the film plane as defined in the scheme at the left side of the figure $(L$ and $M$ axis). The arrows at the right of this scheme indicate the incoming direction of the material flux during the preparation of each part of the sample. The scale bar included in the upper left picture corresponds to $1 \mathrm{~cm}$. 
Since the deposition of GLAD thin films is fully compatible with the use of shadow masks, this technique can be used to deposit $\mathrm{SiO}_{2}$ samples with oxide nanocolumns and gold nanoellipsoids tilted at different angles in selected areas of a given substrate, a situation that is not achievable with polymer host matrixes. ${ }^{[14]}$ Upon gold infiltration, this sample architecture yields multidirectional dichroic materials where distinct zones will respond differently to the polarization of light. An example of the encoding possibilities of laser patterning this type of multidirectional films is shown in Figure $5 c$. The written area extends over three sample zones, each one with a different tilting orientation of the nanocolumns as shown in the scheme. The sequence of pictures shows how the complete laser pattern can only be recovered (picture framed with a red square, where the SPR is identical for the three sample zones) for a given combination of light polarization and sample orientation with respect to the white light beam. Owing to the micrometerscale drawing possibilities of the laser, similar effects could be obtained for micrometer and even sub-micrometer size features. This methodology would enable the use of laser treated $\mathrm{Au} / \mathrm{SiO}_{2}$ GLAD samples for practical applications as optical encoding, engraving, data storage and for anti-counterfeiting protection. In relation with this latter application, written data or codes could only be retrieved if the deposition angle of the columns in every zone is known a priori. Lacking this information, the optical patterns obtained would not correspond to the actually encoded data.

\section{Conclusions}

In this work we have developed a new template-assisted methodology to grow a homogeneous distribution of anisotropic gold NPs in the bulk of optically transparent $\mathrm{SiO}_{2}$ GLAD thin films. Besides its relative simplicity, the method is quite reliable and scalable to large areas. The anisotropic metal NPs present well defined LonSPR and TrSPR modes that can be tuned by adjusting the deposition angle of the GLAD thin films. The asymmetry and high aspect ratio of the metal NPs, together with their tilting along the direction of the $\mathrm{SiO}_{2}$ nanocolumns give rise to a complex dichroic response when turning the samples around both their azimuthal and polar axis. Modeling this optical response with the DDA theory has proved that this complex dichroic behavior is due to both the asymmetric shape of the NPs and their tilted orientation within the intercolumnar space of the $\mathrm{SiO}_{2}$ film. Reshaping the gold NPs by laser irradiation permits the fabrication of dichroic bar codes and, by combining in the same sample regions with different nanocolumns orientation, opens the way to high level encryption processes where the patterned code would only be readable if knowing the exact geometry of nanocolumns in each zone of the the samples.

\section{Experimental Section}

Preparation and Characterization of Dichroic Thin Films: $\mathrm{SiO}_{2}$ GLAD thin films with different nanocolumns tilting angles were prepared by electron evaporation at different zenithal glancing angles, $\alpha$, from $60^{\circ}$ to $85^{\circ}$ (see ref. [25], the scheme in Figure 1 and Figure S5 in the Supporting Information for more details). $\mathrm{SiO}_{2}$ thin films with perpendicular nanocolumns (PN) were fabricated by rotating the substrate placed at a glancing angle during deposition. Gold NPs were grown along the pores of the nanocolumnar $\mathrm{SiO}_{2}$ GLAD thin films by spin-coating on their surface an acetone solution of PMMA containing $\mathrm{HAuCl}_{4}$. After drying, these precursor samples were subsequently heated up to $350^{\circ} \mathrm{C}$. This treatment produced the reduction of the gold species in the precursor samples, the formation of gold NPs and the complete removal of PMMA. The different steps of the process are schematized in Figure 1.

Sample Characterization: Laser treatment conditions to selectively remove the thin film dichroism and experimental details about the characterization methodology of the samples by SEM, GISAXS, and UV-vis transmission are reported in detail in the Supporting Information. A linearly polarized light with the electrical vector parallel to the film surface (i.e., a perpendicular impingement of the beam) was used for the UV-vis analysis. For convenience, to designate the relative orientation of this electrical vector with respect to the film orientation, we have used the same axis frame that the one used to describe the geometrical features of the GLAD thin films (c.f. Figure 1). Thus, $x(y)$-polarized light, as defined in Figure 3, means that the electrical vector is oriented along the $x(y)$ coordinate defined for the films in Figure 1.

\section{Supporting Information}

Supporting Information is available from the Wiley Online Library or from the author.

\section{Acknowledgements}

L.G.-G. and J.P.-B. contributed equally to this work. We thank the Junta de Andalucía (Projects P09-CTS-5189, TEP5283, and FQM-6900) and the Ministry of Economy and Competitiveness (Projects CONSOLIDERCSD 2008-00023, MAT2010-21228, MAT2011-23455, and MAT2010-18447) for financial support. The technical help of A. Mira from the Department of Crystallography of the University of Sevilla (Spain) and J. Perlich from HASYLAB (Germany) is acknowledged. The experiments performed at BW4 in HASYLAB (DESY) for GISAXS analysis were supported by the European Community [Contract RII3-CT-2004-506008 (IASFS)].

Received: July 9, 2012

Revised: October 8, 2012

Published online: November 1, 2012

[1] W.-S. Chang, L. S. Slaughter, B. P. Khanal, P. Manna, E. R. Zubarev, S. Link, Nano Lett. 2009, 9, 1152.

[2] U. M. V. Kreibig, M. Vollmer, Optical Properties of Metal Clusters, Springer, Berlin 1995.

[3] W. A. Murray, W. L. Barnes, Adv. Mater. 2007, 19, 3771.

[4] H. I. El Ahrach, R. Bachelot, A. Vial, G. Lerondel, J. Plain, P. Royer, O. Soppera, Phys. Rev. Lett. 2007, 98, 107402.

[5] E. Fort, C. Ricolleau, J. Sau-Pueyo, Nano Lett. 2003, 3, 65.

[6] R. M. Bakker, H. K. Yuan, Z. T. Liu, V. P. Drachev, A. V. Kildishev, V. M. Shalaev, R. H. Pedersen, S. Gresillon, A. Boltasseva, Appl. Phys. Lett. 2008, 92, 043101.

[7] T. Kitahara, A. Sugawara, H. Sano, G. Mizutani, J. Appl. Phys. 2004, 95, 5002.

[8] H. Mertens, A. Polman, Appl. Phys. Lett. 2006, 89, 211107.

[9] M. Cavallini, C. Albonetti, F. Biscarini, Adv. Mater. 2009, 21, 1043.

[10] P. Greco, M. Cavallini, P. Stoliar, S. D. Quiroga, S. Dutta, S. Zacchini, M. C. Iapalucci, V. Morandi, S. Milita, P. G. Merli, F. Biscarini, J. Am. Chem. Soc. 2008, 130, 1177.

[11] T. Wenzel, J. Bosbach, A. Goldmann, F. Stietz, F. Trager, Appl. Phys. B 1999, 69, 513 . 
[12] M.-C. Garcia-Gutierrez, A. Linares, J. J. Hernandez, D. R. Rueda, T. A. Ezquerra, P. Poza, R. J. Davies, Nano Lett. 2010, 10, 1472.

[13] Y. Dirix, C. Bastiaansen, W. Caseri, P. Smith, Adv. Mater. 1999, 11, 223.

[14] J. Pérez-Juste, B. Rodríguez-González, P. Mulvaney, L. Liz-Marzán, Adv. Funct. Mater. 2005, 15, 1065.

[15] M. M. Hawkeye, M. J. Brett, J. Vac. Sci. Technol., A 2007, 25, 1317.

[16] M. J. Brett, M. M. Hawkeye, Science 2008, 319, 1192.

[17] R. Alvarez, L. Gonzalez-Garcia, P. Romero-Gomez, V. Rico, J. Cotrino, A. R. Gonzalez-Elipe, A. Palmero, J. Phys. D: Appl. Phys. 2011, 44, 385302.

[18] J. R. Sanchez-Valencia, I. Blaszczyk-Lezak, J. P. Espinos, S. Hamad, A. R. Gonzalez-Elipe, A. Barranco, Langmuir 2009, 25, 9140.

[19] H. Vankranenburg, C. Lodder, Mater. Sci. Eng., R 1994, 11, 295.

[20] J. R. Sanchez-Valencia, J. Toudert, A. Borras, C. Lopez-Santos, A. Barranco, I. Ortega Feliu, A. R. Gonzalez-Elipe, Plasmonics 2010, 5, 241

[21] J. R. Sanchez-Valencia, J. Toudert, A. Borras, A. Barranco, R. Lahoz, G. F. de la Fuente, F. Frutos, A. R. Gonzalez-Elipe, Adv. Mater. 2011, 23,848 .
[22] L. Gonzalez-Garcia, I. Gonzalez-Valls, M. Lira-Cantu, A. Barranco A. R. Gonzalez-Elipe, Energy Environ. Sci. 2011, 4, 3426.

[23] N. J. Gerein, M. D. Fleischauer, M. J. Brett, Sol. Energ. Mater. Sol. Cells 2010, 94, 2343.

[24] L. Gonzalez-Garcia, A. Barranco, A. Munoz-Paez, A. R. Gonzalez-Elipe, M. C. Garcia-Gutierrez, J. J. Hernandez, D. R. Rueda, T. A. Ezquerra, D. Babonneau, ChemPhysChem 2010, 11, 2205.

[25] L. Gonzalez-Garcia, G. Lozano, A. Barranco, H. Miguez, A. R. Gonzalez-Elipe, J. Mater. Chem 2010, 20, 6408.

[26] A. C. Van Popta, J. Cheng, J. C. Sit, M. J. Brett, J. Appl. Phys. 2007 102, 013517.

[27] D. Vaughan, X-Ray Rata Booklet, Lawrence Berkeley National Laboratory, Berkeley, CA 2009.

[28] H. Devoe, J. Chem. Phys. 1964, 41, 393.

[29] E. M. Purcell, C. Pennypac, Astrophys J. 1973, 186, 705

[30] B. T. Draine, P. J. Flatau, J. Opt. Soc. Am. A 1994, 11, 1491.

[31] B. T. Draine, P. J. Flatau, J. Opt. Soc. Am. A 2008, 25, 2693.

[32] B. T. Draine, P. J. Flatau, User Guide for the Discrete Dipole Approximation Code DDSCAT 7.1 2010, arXiv:1002.1505. 